\title{
Exposición a la Violencia y Riesgo Suicida en Adolescentes Colombianos
}

\section{Exposure to Violence and Suicide Risk in Colombian Adolescents}

\author{
Yuly Suárez \\ Grupo de Investigación Psicología y Salud, Universidad del Magdalena, Colombia \\ Dayana Restrepo \\ Grupo de Investigación en Psicología, Universidad del Norte, Colombia \\ Carmen Caballero \\ Grupo de Investigación Psicología y Salud, Universidad del Magdalena, Colombia \\ Jorge Palacio \\ Grupo de Investigaciones en Desarrollo Humano (GIDHUM), Colombia
}

Recibido (29 de agosto de 2017) Aceptado (1 de junio de 2018)

\begin{abstract}
Resumen
Se analizó la asociación entre el riesgo suicida con la exposición a la violencia en la casa, calle, colegio, televisión en adolescentes de Santa Marta (Colombia). Se evaluó una muestra de 210 adolescentes entre 12-19 años $(\mathrm{M}=14.6 \mathrm{DE}=1.38)$. Los sujetos respondieron la Escala de Riesgo Suicida de Plutchik, además de la Escala de Exposición a la Violencia. Se encontró asociación bivariada con la exposición a la violencia en la casa. En un modelo de regresión logística ajustado el apoyo de padres padres/hermanos/amigos $(\mathrm{OR}=0.213 \mathrm{IC} 95 \%=0.70-0649)$ es un factor protector para el riesgo suicida, mientras el género femenino $(\mathrm{OR}=5.397 \mathrm{IC} 95 \%=2.541-11.460)$, exposición a la violencia en la casa $(\mathrm{OR}=2.334 \mathrm{IC} 95 \%=1.161-4.298)$ y dificultades de ingreso a la educación ( $\mathrm{OR}=3.257 \mathrm{IC} 95 \%=1.013-10.467)$ son factores de riesgo. Se concluye que mujeres adolescentes, víctimas de violencia en el hogar y con problemas para acceder a la educación básica tienen mayor probabilidad de presentar riesgo suicida.

Palabras Clave: violencia, suicidio, adolescentes
\end{abstract}

\begin{abstract}
The association between suicide risk and exposure to violence in the home, street, school, television in adolescents of Santa Marta (Colombia) was analyzed. A sample of 210 adolescents aged 12-19 years $(\mathrm{M}=$ 14.6 SD = 1.38) was evaluated. The subjects responded to the Plutchik Suicide Risk Scale and the Violence Exposure Scale. We found a bivariate association with exposure to violence in the home. In an adjusted logistic regression model the support of parents / siblings / friends (OR $=0.213$ CI 95\% $=0.70-0649)$ is a protective factor for suicidal risk, while female gender $(\mathrm{OR}=5.39795 \% \mathrm{CI}=2.541-11.460)$, exposure to violence in the home $(\mathrm{OR}=2.344,95 \% \mathrm{CI}=1.161-4.298)$ and difficulties in entering education $(\mathrm{OR}=$ $3.257,95 \% \mathrm{CI}=1013-10.467)$ are risk factors. It is concluded that adolescent women, victims of domestic violence and with problems to access basic education are more likely to present suicidal risk.

Keywords: violence, suicide, adolescents
\end{abstract}

Correspondencia: Yuly Suárez, email: ysuc91@gmail.com, Carrera 32, No 22-08, Santa Marta, Colombia (470004).

*Investigación apoyada por la Gobernación del Magdalena (Colombia) y Colciencias- Formación de Capital Humano de Alto Nivel para las Regiones-

Convocatoria Regalías Departamento Del Magdalena 672 de 2014. 


\section{Introducción}

La adolescencia es una de las etapas del desarrollo con mayor vulnerabilidad a la exposición directa o indirecta de la violencia (Dapieve \& Dalvosco, 2017). Algunos estudios identifican que las auto laceraciones, el abuso de alcohol y drogas, los problemas de conducta, el bajo rendimiento académico, la depresión, las ideaciones y comportamientos suicidas son resultados adversos de un sujeto que ha estado expuesto a la violencia (Ford, Hartman, Hawke, \& Chapman, 2008; Ford, Elhai, Connor, y Frueh, 2010; Mrug y Windle, 2010; Swahn, Gaylor, Bossarte, y Dulmen, 2010; Vermeiren, Ruchkin, Leckman, Deboutte y Schwab-Stone, 2002).

Generalmente, los análisis de la exposición a la violencia en la adolescencia tienen la limitación de referirse a un sólo contexto o situación, por ejemplo, la exposición a la violencia en la comunidad (Brook, Brook \& Whiteman, 2007; Guedes, García-Moreno, y Bott, 2014; Mels \& Fernández, 2015), en la televisión (Rojas, 2008), en la familia (Gámez-Guadix \& Calvete, 2012; Piedrahita, Elvira, Martinez \& Vinazco, 2007) o en la escuela (Beck, Arif, Paumier y Jacobsen, 2016; Hoyos, Aparicio \& Córdoba, 2005). De allí que ha quedado pendiente analizar la exposición a la violencia en diferentes contextos simultáneamente y revisar su relación con los comportamientos suicidas de los adolescentes.

Por esto se propone la investigación con adolescentes expuestos a violencia en diferentes contextos, para determinar su asociación con el riesgo de suicido. Específicamente se hace relevante esta investigación en un contexto como el de Colombia, un país afectado por el conflicto armado durante los últimos 50 años y con estadísticas alarmantes de violencia: 26.985 casos de violencia intrafamiliar de los cuales 10.435 casos correspondieron a violencia contra niños, niñas y adolescentes, 1.651 casos de violencia contra la población adulta mayor, 14.899 casos de violencia entre otros familiares y 11.585 homicidios (Instituto de Medicina Legal y Ciencias Forenses, 2015a; 2015b).

\section{Contextos de Exposición a la Violencia y Riesgo Suicida}

- Violencia en el Hogar: Un importante cuerpo de investigaciones ha identificado a la violencia en el hogar como un claro factor de riesgo para el suicidio durante el curso de la vida (Cha \& Nock, 2009; Hooven, Nurius, Logan-Greene \& Thompson, 2012). Se ha demostrado cómo la violencia en el hogar genera sentimientos de tristeza, rabia, desconfianza, confusión, culpabilidad, vergüenza y pensamientos de muerte en los niños y adolescentes (Ramírez, 2006). Los efectos psicológicos de estas experiencias pueden rastrearse aún décadas después, a manera de síntomas de estrés postraumático, desórdenes depresivos, trastornos de ansiedad, ideas e intentos suicidas en individuos expuestos a la violencia en sus hogares (Devries, Mak, Bacchus, Child, Falder, Petzold, Astbury \& Watts, 2013; Margolin \& Vickerman, 2007)

- Violencia en la Comunidad: Al igual que sucede con los ambientes familiares conflictivos, las investigaciones señalan a los entornos comunitarios con elevados índices de violencia como grandes obstáculos que dificultan el desarrollo psicológico y emocional de los niños -adolescentes (Amone-P'Olak, Garnefski \& Kraaij, 2007; Brook et al., 2007; Cole, 2014; Mejia, Kliewer \& Williams, 2006; Raver, 2004; Wadsworth \& Berger, 2006). Los menores que crecen expuestos a violencia en su entorno comunitario encuentran menores posibilidades para su desarrollo social y personal: vulnerabilidad a ser víctimas de la violencia callejera, escasas oportunidades educativas y exposición permanentemente a modelos de vida negativos (Raver, 2004; Wadsworth \& Berger, 2006; Wadsworth \& Compas, 2002). La exposición a la violencia en la comunidad incluye varias formas de victimización para los adolescentes, entre ellas: ser blanco directo de robos, tiroteos, peleas o testigo de dichos actos cometidos hacia otros.

Esta exposición directa e indirecta a la violencia en la comunidad ha sido asociada con aparición de síntomas de estrés, ansiedad y depresión que deterioran la salud emocional del adolescente y por ende su sano desarrollo (Chen, 2010; Hewitt et al., 2014; Mrug \& Windle, 2010). De manera puntual, la violencia comunitaria dirigida contra un miembro de la familia, amigo cercano o alguien de igual filiación ideológica al adolescente, se ha encontrado asociada a síntomas depresivos de rango clínico y con ideas de suicidio entre los adolescentes (Duncan \& Hatzenbuehler, 2014; Lambert, Boyd, Cammack \& Ialongo, 2012).

- Violencia en la Escuela: Mientras, existe una relación directa entre las experiencias de violencia en la escuela y el comportamiento suicida en adolescentes. Los hallazgos de estudios longitudinales indican un elevado riesgo de ideación e intentos suicidas en aquellos adolescentes que han sido expuestos a violencia prolongada en la escuela, especialmente si el adolescente ha sido la víctima de tales actos de violencia (Bonanno \& Hymel, 2010; Klomek, Sourander \& Gould, 2010; Takizawa, Maughan \& Arseneault, 2014; Waseem, Ryan, Foster \& Peterson, 2013).

La forma más frecuente de violencia en la escuela y de mayor prevalencia en el mundo ha recibido el nombre de "bullying" o "maltrato entre iguales". Ésta se refiere a una serie de agresiones por parte de un estudiante o grupo de estudiantes hacia otro, con la característica principal que es sostenida en el tiempo (Debarbieux, 2003). La violencia escolar involucra varios tipos de agresiones, éstas pueden ser físicas como golpear, patear, arrebatar objetos o agresiones verbales en forma de burlas, sobrenombres, amena- 
zas e incluso otras formas de agresión como la exclusión social del adolescente, quien es ignorado o no le permiten participar, entre otras (Bonanno \& Hymel, 2010; Hinduja \& Patchin, 2010; Hoyos, et al., 2005; Hoyos, Llanos \& Mackenzie, 2012; Waseem, Ryan, Foster \& Peterson, 2013). Las víctimas de estos tipos de violencia escolar reportan sentimientos de invalidez, inadecuación, desesperanza y soledad, todos los cuales se han visto directamente asociados a la aparición de pensamientos y conductas suicidas (Bonanno \& Hymel, 2010; Klomer et al., 2010; Waseem, Ryan, Foster \& Peterson, 2013).

- Violencia en la televisión: La televisión como medio masivo de influencia tiene el potencial de afectar negativamente muchos aspectos del desarrollo de los adolescentes incluyendo las creencias, comportamientos hacia la violencia y el comportamiento suicida (Hawton, Saunders \& O'Connor, 2012; Ray \& Jat, 2010; Sandoval Escobar, 2006; Strenziok, Krueger, Pulaski, Openshaw, Zamboni, Van der Meer \& Grafman, 2010).

Los estudios demuestran cómo la exposición intensiva y excesiva a la violencia en la televisión u otras formas de exposición como en videojuegos se asocia a la depresión y tendencia suicida (Messias, Castro, Saini, Usman \& Peeples, 2011). Incluso al ser el suicidio una forma de violencia interpersonal, la exposición a estas formas de violencia se ha encontrado relacionada con el suicidio, específicamente se conoce que existe una relación entre las noticias y reportajes sobre suicidios que transmiten en la televisión y el incremento en las tasas de suicido entre la población adolescente (Chen et al., 2010; Dunlop, More \& Romer, 2011; Sandoval Escobar, 2006; Haw, Hawton, Niedzwiedz \& Platt, 2013; Pirkis \& Blood, 2010). Es decir que los mecanismos psicológicos como el referenciamiento social, contagio, imitación, sugestión, identificación pueden activarse, luego de una exposición directa o indirecta a un suicidio, sea visto en la televisión, en la comunidad o en el círculo social cercano del adolescente (Yip et al., 2006; Pirkis \& Blood, 2010; Chen et al., 2010).

Teniendo en cuenta el vínculo existente entre la exposición a la violencia en la familia, en la escuela, en la comunidad, en la televisión y los comportamientos suicidas en la adolescencia es válido preguntarse ¡cuál es la asociación entre la exposición a la violencia en la casa, la calle, el colegio, la televisión y el riesgo suicida en una muestra de adolescentes de Santa Marta, Colombia.

\section{Método}

\section{Participantes}

Se seleccionó intencionalmente una muestra de 210 adolescentes con edades entre 12 y 19 años (Media $=14.6$ Desviación Típica $=1.38), 57.1 \%$ fueron mujeres y $42.9 \%$ hombres, escolarizados de $8^{\circ}$ a $11^{\circ}$ grado en un colegio público de Santa Marta (norte de Colombia).

\section{Instrumentos}

Cuestionario sociodemográfico. Los autores diseñaron un cuestionario con opciones de respuesta dicotómicas, que explora características como género, edad, vida con padres, vida con madre, dificultades de ingreso a la educación, dificultades para continuar con los estudios, dificultades de acceso a salud, trabajo y apoyo de padres/hermanos/amigos.

Escala de Riesgo Suicida (Plutchik, Van Praga, Contey Picard, 1989) adaptada para España por Rubio et al. (1998) evalúa en 15 ítems el riesgo suicida a partir de factores de riesgo; es un instrumento con opción de respuesta dicotómica (sí/no). Presenta un alfa de cronbach de 0.89 , sensibilidad y especificidad del $88 \%$ para discriminar entre individuos (Rubio et al., 1998). Ver tabla 1.

Escala de Exposición a la Violencia (Orue, 2010): evalúa en 21 ítems la exposición a la violencia física, verbal o amenaza de forma directa (victima) e indirecta (observador) en diferentes contextos: colegio, calle, casa y televisión. Sus respuestas son en una escala Likert de 0 (nunca) a 4 (todos los días). De acuerdo a Orue (2010) presenta un alfa de cronbach de 0.80 para la exposición en el colegio, 0.80 en la calle, y 0.86 en la casa. Ver tabla 1.

Tabla 1. Análisis de fiabilidad de instrumentos en el presente estudio

\begin{tabular}{llllllll}
\hline Variables & & Ítems & Media & Desv. Típica & Mín & Máx & $\begin{array}{l}\text { Consistencia } \\
\text { interna* }\end{array}$ \\
\hline Exposición a la & En colegio & 6 & 8.02 & 4.348 & 0 & 21 & 0.734 \\
Violencia & En calle & 6 & 8.65 & 4.240 & 0 & 24 & 0.713 \\
& En casa & 6 & 6.06 & 4.924 & 0 & 23 & 0.795 \\
& En televisión & 3 & 8.14 & 3.165 & 0 & 16 & 0.675 \\
\hline Riesgo Suicida & & 15 & 4.28 & 3.278 & 0 & 12 & $0.799 * *$ \\
\hline
\end{tabular}

Nota. * Alfa de Crombach, ** KR20 


\section{Procedimiento}

En esta investigación se tuvo en cuenta la declaración de Helsinki (Asociación Mundial de Medicina) y la Ley 1090 de septiembre 6 de 2006 (Congreso de la República Colombiana) para la investigación con humanos y para la reglamentación del ejercicio de la psicología en el país. En el abordaje de los participantes se conservó el derecho al anonimato, la participación voluntaria, el retiro en cualquier momento de la investigación, la devolución de resultados $y$, en caso de ser necesario, la atención psicológica de emergencia al adolescente.

Luego de explicar los objetivos y la finalidad del estudio, los colegios autorizaron la investigación con los estudiantes. Se entregó a los participantes el consentimiento informado con los detalles de la investigación para el conocimiento y autorización de padres o tutores. Los estudiantes que devolvieron el documento autorizado por los padres recibieron el asentimiento para su firma, donde expresaron su participación voluntaria en el estudio. Luego se aplicaron los instrumentos. Posteriormente, se segmentó la base de datos de acuerdo al puntaje en la escala de riesgo suicida para definir los grupos con y sin riesgo suicida.

\section{Análisis}

Como herramienta para el análisis de la información se utilizó el software de análisis estadístico para las ciencias sociales-SPSS 23. Para el presente análisis se tomó como variable dependiente el riesgo suicida y como variables independientes se consideraron la exposición a la violencia en la casa, calle, colegio y televisión, además de algunas variables sociodemográficas. Todas las variables se dicotomizaron cuando no era la condición natural.

Inicialmente se calcularon las asociaciones bi-variadas a través de razones de disparidad (OR) con intervalo de confianza del 95\% (IC95\%), se ingresaron al modelo de regresión logística no condicionada las variables con asociaciones mayores a 0.2 , se controlaron las posibles variables extrañas, aplicando las recomendaciones de Greenland y la bondad del ajuste en la prueba de Hosmer-Lemeshow para evaluar el mejor modelo.

Tabla 2. Frecuencias del riesgo suicida y la exposición a la violencia en adolescentes

\begin{tabular}{|c|c|c|c|c|}
\hline Variables & & Categorías & $\%$ & $\mathrm{~F}$ \\
\hline \multirow{2}{*}{ Riesgo Suicida } & & Sin riesgo & 68.6 & 144 \\
\hline & & Con riesgo & 31.4 & 66 \\
\hline \multirow{18}{*}{ Tipo de Exposición a la Violencia } & \multirow{2}{*}{ En colegio } & Sin exposición & 58.1 & 122 \\
\hline & & Con exposición & 41.9 & 88 \\
\hline & \multirow{2}{*}{ En calle } & Sin exposición & 53.8 & 113 \\
\hline & & Con exposición & 46.2 & 97 \\
\hline & \multirow{2}{*}{ En casa } & Sin exposición & 61.0 & 128 \\
\hline & & Con exposición & 39.0 & 82 \\
\hline & \multirow{2}{*}{ La TV } & Sin exposición & 50 & 50 \\
\hline & & Con exposición & 50 & 50 \\
\hline & \multirow{2}{*}{ Directa } & Sin exposición & 54.8 & 115 \\
\hline & & Con exposición & 45.2 & 95 \\
\hline & \multirow{2}{*}{ Indirecta } & Sin exposición & 55.7 & 117 \\
\hline & & Con exposición & 44.3 & 93 \\
\hline & \multirow{2}{*}{ Física } & Sin exposición & 55.7 & 117 \\
\hline & & Con exposición & 44.3 & 93 \\
\hline & \multirow{2}{*}{ Amenazas } & Sin exposición & 54.8 & 115 \\
\hline & & Con exposición & 45.2 & 95 \\
\hline & \multirow{2}{*}{ Verbal } & Sin exposición & 50.5 & 106 \\
\hline & & Con exposición & 49.5 & 104 \\
\hline
\end{tabular}




\section{Resultados}

El análisis descriptivo indica un 31.4\% de adolescentes con riesgo suicida. Al respecto de la exposición a la violencia en diferentes contextos, el $41.9 \%$ reportó exposición a la violencia en el colegio, el 53.8\% exposición a la violencia en la calle, el $61 \%$ exposición a la violencia en la casa, y el $50 \%$ exposición a la violencia en la televisión. En general, la experiencia de la violencia en los adolescentes participantes se representó en el $45.2 \%$ de exposición directa a la violencia y un $44.3 \%$ de exposición indirecta a la violencia. Asimismo, las tipologías de la violencia rastreadas se evidenciaron en un $44.3 \%$ de exposición a la violencia física, un $45.2 \%$ de exposición a la violencia amenazas, y un $49.5 \%$ de violencia verbal (ver tabla 2).

A través del análisis de contingencia se observó que adolescentes con riesgo suicida presentan un $12.4 \%$ de exposición a la violencia en el colegio, un $16.7 \%$ de exposición a la violencia en la calle, $17.1 \%$ de exposición a la violencia en la casa y $15.2 \%$ de exposición a la violencia en la televi- sión. Por otra parte, el $11 \%$ no vive con el padre, $1.9 \%$ no vive con la madre, $3.8 \%$ presenta dificultades de ingreso a la educación, $7.1 \%$ dificultades de acceso a salud, $6.2 \%$ dificultades de acceso a medicamentos, $27.6 \%$ dificultades para continuar los estudios, el $2.9 \%$ trabaja y $5.2 \%$ no tiene apoyo de padres/hermanos/amigos (ver tabla 3).

El análisis bi-variado mostró que el riesgo suicida en adolescentes se asocia significativamente a la exposición a la violencia en la casa $(\mathrm{OR}=2,330 \mathrm{IC} 95 \%=1.284-4.228)$, el género $(\mathrm{OR}=4.151 \mathrm{IC} 95 \%=2.114-8.151)$, y el apoyo de padres/hermanos/amigos (OR=0,255 IC95\% $=0.094-0.693)$. Ver tabla 4.

Tabla 3. Contingencia entre el riesgo suicida, exposición a la violencia en adolescentes y variables sociodemográficas

\begin{tabular}{|c|c|c|c|}
\hline \multirow{2}{*}{\multicolumn{2}{|c|}{ Variables }} & \multicolumn{2}{|c|}{ Riesgo Suicida \% } \\
\hline & & Sin Riesgo Suicida & Con Riesgo Suicida \\
\hline \multirow{2}{*}{ Género } & Femenino & 32.4 & 24.8 \\
\hline & Masculino & 36.2 & 6.7 \\
\hline \multirow{2}{*}{ Violencia colegio } & Sin exposición & 3.0 & 19.0 \\
\hline & Con exposición & 29.5 & 12.4 \\
\hline \multirow{2}{*}{ Violencia calle } & Sin exposición & 39.0 & 14.8 \\
\hline & Con exposición & 29.5 & 16.7 \\
\hline \multirow{2}{*}{ Violencia casa } & Sin exposición & 46.7 & 14.3 \\
\hline & Con exposición & 21.9 & 17.1 \\
\hline \multirow{2}{*}{ Violencia TV } & Sin exposición & 33.8 & 16.2 \\
\hline & Con exposición & 34.8 & 15.2 \\
\hline \multirow{2}{*}{ Vivo con mi padre } & No & 17.1 & 11.0 \\
\hline & Sí & 51.4 & 20.5 \\
\hline \multirow{2}{*}{ Vivo con mi madre } & No & 3.3 & 1.9 \\
\hline & Sí & 65.2 & 29.5 \\
\hline \multirow{2}{*}{ Dificultades ingreso educación } & No & 64.8 & 27.6 \\
\hline & Sí & 3.8 & 3.8 \\
\hline \multirow{2}{*}{ Dificultades continuar estudios } & No & 4.3 & 3.8 \\
\hline & Sí & 64.3 & 27.6 \\
\hline \multirow{2}{*}{ Dificultades acceso salud } & No & 55.7 & 24.3 \\
\hline & Sí & 12.9 & 7.1 \\
\hline \multirow{2}{*}{ Dificultades acceso medicamentos } & No & 58.1 & 25.2 \\
\hline & Sí & 10.5 & 6.2 \\
\hline \multirow{2}{*}{ Trabaja } & No & 66.2 & 28.6 \\
\hline & Sí & 2.4 & 2.9 \\
\hline \multirow{2}{*}{ Apoyo de padres/hermanos/amigos } & No & 3.3 & 5.2 \\
\hline & Sí & 65.2 & 26.2 \\
\hline
\end{tabular}


Tabla 4. Asociación bivariada entre el riesgo suicida y la exposición a la violencia en adolescentes

\begin{tabular}{|c|c|c|c|c|c|c|c|c|}
\hline & \multirow[t]{2}{*}{ B } & \multirow{2}{*}{$\begin{array}{l}\text { Error } \\
\text { estándar }\end{array}$} & \multirow{2}{*}{ Wald } & \multirow[t]{2}{*}{ gl } & \multirow[t]{2}{*}{ Sig. } & \multirow{2}{*}{$\begin{array}{l}\text { Exp } \\
\text { (B) }\end{array}$} & \multicolumn{2}{|c|}{$\begin{array}{l}\text { 95\% C.I. para } \\
\text { EXP (B) }\end{array}$} \\
\hline & & & & & & & Inferior & Superior \\
\hline Exposición violencia casa & .846 & .304 & 7.743 & 1 & .005 & 2.330 & 1.284 & 4.228 \\
\hline Exposición violencia calle & .401 & .299 & 1.803 & 1 & .179 & 1.493 & .832 & 2.681 \\
\hline Exposición violencia colegio & -.151 & .303 & .249 & 1 & .618 & .860 & .475 & 1.557 \\
\hline Exposición violencia TV & -.088 & .297 & .088 & 1 & .766 & .915 & .511 & 1.640 \\
\hline Género & -1.423 & .344 & 17.095 & 1 & .000 & 4.151 & 2.114 & 8.151 \\
\hline Vive con padre & -.473 & .332 & 2.155 & 1 & .142 & .623 & .331 & 1,172 \\
\hline Vive con madre & -.233 & .645 & .131 & 1 & .718 & .792 & .224 & 2.805 \\
\hline Dificultades ingreso a la educación & .852 & .524 & 2.645 & 1 & .104 & 2.345 & .840 & 6.549 \\
\hline Dificultad para continuar estudios & -.727 & .511 & 2.027 & 1 & .155 & .483 & .178 & 1.315 \\
\hline Dificultades de acceso salud & .243 & .363 & .446 & 1 & .504 & 1.275 & .626 & 2.597 \\
\hline Trabajo & 1.022 & .625 & 2.677 & 1 & .102 & 2.780 & .817 & 9.462 \\
\hline Apoyo de padres/hermanos/amigos & -1.365 & .509 & 7.183 & 1 & .007 & .255 & .094 & .693 \\
\hline
\end{tabular}

Tabla 5. Modelo Regresión Logística Ajustado del Riesgo Suicida en Adolescentes

\begin{tabular}{lcccccccc}
\hline & & & & & \multicolumn{5}{c}{$95 \%$ C.I. para EXP(B) } \\
& $\mathrm{B}$ & Error estándar & Wald & Gl & Sig. & Exp(B) & Inferior & Superior \\
\hline Género & -1.686 & .304 & 19.249 & 1 & .000 & 5.397 & 2.541 & 11.460 \\
Apoyo de padres/hermanos/amigos & -1.547 & .569 & 7.398 & 1 & .007 & .213 & .070 & .649 \\
Exposición violencia casa & .804 & .334 & 5.796 & 1 & .016 & 2.234 & 1.161 & 4.298 \\
Dificultades de ingreso educación & -1.181 & .596 & 3.298 & 1 & .047 & 3.257 & 1.013 & 10.467 \\
\hline
\end{tabular}

Nota. Bondad del ajuste de Hosmer-Lemeshow, $\mathrm{X} 2=3.542, \mathrm{gl}=5, \mathrm{p}=0.617$

Al modelo se ingresaron variables con valores de $\mathrm{p}>0.20$, éstas fueron: exposición a la violencia en la casa, exposición a la violencia en la calle, exposición a la violencia en el colegio, exposición a la violencia en la tv, género, edad, vida con padre, vida con madre, dificultades de ingreso a la educación, dificultades para continuar con los estudios, dificultades de acceso a la salud, trabajo y apoyo de padres/hermanos/amigos. El modelo de regresión mostró asociaciones a un valor $\mathrm{p}<0.05$ entre el riesgo suicida $\mathrm{y}$ el género $(\mathrm{OR}=5.397 \mathrm{IC} 95 \%=2.541-11.460)$, apoyo de padres/hermanos/amigos $(\mathrm{OR}=0.213 \mathrm{IC} 95 \%=0.70-0649)$, exposición a la violencia en la casa $(\mathrm{OR}=2,334 \mathrm{IC} 95 \%=1.161$ 4.298), y dificultades de ingreso a la educación $(\mathrm{OR}=3.257$ IC95\%=1.013-10,467). De acuerdo al Hosmer-Lemeshow, el modelo presenta bondad de ajuste $\mathrm{p}>0.05$ (ver tabla 4 ).

\section{Discusión}

El estudio de la exposición a la violencia en la casa, calle, colegio, televisión y el riesgo suicida se realizó en un contexto de vulnerabilidad caracterizado por las dificultades para ingresar al sistema educativo, continuar con estudios avanzados, acceder al sistema de salud, acceder a medicamentos, trabajo infanto-juvenil, una representativa formación educativa de padres en secundaria, incluso primaria, y acceso restringido a servicios públicos básicos para la calidad de vida de la población. Además, la lectura del contexto social sugiere que existen factores que alteran la salud mental, relacionados con el consumo de sustancias psicoactivas, impulsividad, soledad, pesimismo, desesperanza, síntomas depresivos, ideas e intentos de suicidio, o 
historia familiar de intentos de suicido. Las interacciones de estos factores psicosociales configuran un riesgo que puede afectar la supervivencia de los adolescentes a través del riesgo suicida.

Al analizar las asociaciones a través de un modelo de regresión logística ingresando variables de carácter psicosocial, se identificó como factores de riesgo el género, la exposición a la violencia en la casa y las dificultades de ingreso a la educación. Al respecto, es bien conocido que el género femenino presenta mayormente síntomas de ansiedad, depresión y riesgo suicida en comparación con los hombres (Chen, 2010; Daine, Hawton, Singaravelu, Stewart, Simkin \& Montgomery, 2013; Lowry, Crosby, Brener, y Kann, 2014; Lambert, Boyd, Cammack \& Ialongo, 2012; Waldrop, Hanson, Resnick, Kilpatrick, Naugle \& Saunders, 2007). Incluso aunque el suicidio consumado sea más frecuente en hombres que en mujeres (Navarro-Gómez, 2017). Estas diferencias algunas veces son explicadas por el procesamiento de la información emocional particular de género, el patrón de gestión emocional propio de la cultura, por el acceso a métodos más lesivos en hombres y por estrategias activas recurrentes para afrontar dificultades, menos frecuente en masculinos (Suárez \& Wilches, 2015).

Asimismo, se encontró que experiencias victimizantes en la casa se asocian al riesgo suicida, resultados coherentes con investigaciones que señalan al desajuste emocional generalizado producto de ideas de suicidio en niños y adolescentes que han crecido expuestos a circunstancias de violencia en su entorno familiar (Gámez-Guadix \& Calvete, 2012; Piedrahita et al., 2007); esta exposición a la violencia en la casa - sea como observador y/o víctima en sus diversas tipologías agresión física, agresión verbal y amenazas-, tiene un rol preponderante en conductas de riesgo para la salud (Devries et al., 2013; Hoveen, Nurius, Logan-Greene \& Thompson, 2012; Klomek et al., 2010) e interrumpen el desarrollo emocional del adolescente (Castellvi, et al, 2017; Hooven et al., 2012; Rajalin, Hirvikoski \& Jokinen, 2013). Sin embargo, la exposición a la violencia en el colegio, la calle, o la televisión no amenaza la supervivencia en estos adolescentes. Aunque algunos estudios también señalan a la violencia en la comunidad (Duncan y Hatzenbuehler, 2014; Lambert et al., 2012), en la escuela (Klomek et al., 2010; Takizawa et al., 2014) y la exposición intensiva/excesiva a la violencia en la televisión (Messias et al., 2011) como factores de riesgo para muerte por suicidio.

Por otra parte, los resultados apuntan a que dificultades de ingreso a la educación se asocian al riesgo suicida, en concordancia con las evidencias que reconocen a las condiciones psicosociales adversas especialmente en mujeres se relacionan con el intento de suicidio (Mandal, y Zalewskan, 2010; 2012), mientras, otros estudios indican que la experiencia de la violencia en la casa, sumado a la falta de vivienda y otras características socioeconómicas, se relacionaban con comportamientos suicidas (Massey, Compton \& Kaslow, 2014). Esta perspectiva del suicidio desde las condiciones económicas muestra cómo la pobreza y desigualdad funcionan como determinantes sociales en salud (Campo-Arias \& Herazo, 2015).

También se identificó como factor protector el apoyo de padres/hermanos/amigos, lo que indica la relevancia de los factores relacionados con la experiencia social como es la percepción de apoyo de la familia y los pares, tan importantes en el vínculo afectivo adolescencial proporcionando seguridad para afrontar los retos, demandas y amenazas, vinculado profundamente con la salud mental/ psicopatología (Lipschitz, Yen, Weinstock \& Spirito, 2012; Pardo, Pineda, Carrillo \& Castro, 2006).

Si la violencia es de tipo físico puede existir riesgo para intentos de suicidio en adolescentes (Espinoza-Gómez, Zepeda-Pamplona, Bautista-Hernández, HernándezSuárez, Newton-Sánchez \& Placensia-García, 2010), si las agresiones son verbales sistemáticas y de amenazas pueden existir graves repercusiones psicológicas y aunque constituyen actos inmateriales de violencia se ha encontrado que atentan por igual contra la valía personal y el ajuste emocional del adolescente, como si se tratara de agresiones físicas y por tanto pueden propiciar el desarrollo de conducta suicida (Hagan, \& Joiner, 2016; Savin-Williams, 1994; Borowsky, Taliaferro \& McMorris, 2013). Particularmente, respecto a las amenazas, la literatura indica que tanto ser víctima directa de amenazas como observar que otro es amenazado son experiencias adversas equivalentes para el adolescente, que deterioran su salud mental (Wang, Sareen, Afifi, Bolton, Johnson \& Bolton, 2015).

En conclusión, es claro que la exposición a la violencia constituye un riesgo elevado para manifestar el comportamiento suicida en la adolescencia, pero si los jóvenes expuestos a violencia y a dificultades para acceder a la educación no evidencian este tipo de comportamiento, se puede asumir para este grupo de jóvenes que es porque existen factores protectores representados sobre todo en las fuertes redes de apoyo de sus padres, familiares y amigos (Bonanno \& Hymel, 2010; Ozer, 2005; Rigby \& Slee, 1999). Esta periferia del riesgo suicida sugiere que siempre será indispensable la oportuna intervención en mujeres adolescentes, víctimas de violencia en el hogar, con problemas para acceder a la educación básica y poco apoyo de padres/ hermanos/amigos.

No se descarta que existan otros factores relevantes como los estilos de crianza (Nicolaev, Barajova \& Petunova, 2016) y las habilidades emocionales que podrían ser importantes en la salud mental de los adolescentes especialmente expuestos a la violencia en la casa, colegio, calle o televisión al incidir en el ajuste psicológico duradero, a pesar de 
circunstancias adversas (Czyz, Horwitz, Eisenberg, Kramer \& King, 2013; Dasgupta \& Hazra, 2011; Dunlop, More \& Romer, 2011; Jacobson, Marrocco, Kleinman \& Gould, 2011; Kim \& Cicchetti, 2010; Pisani, Wyman, Petrova, Schmeelk-Cone, Goldston, Xia \& Gould, 2013; Suárez, Restrepo \& Caballero, 2016).

Esta investigación presenta limitaciones para su generalización por estudiar un contexto particular de Colombia, no obstante, permite establecer indicios de los factores más relacionados con la violencia en un contexto de restablecimiento o recuperación psicosocial. Es necesario explorar con más detalle la asociación de estas variables e ir direccionando la promoción de una cultura para la paz y la educación para la convivencia pacífica focalizada en la prevención del suicido desde una perspectiva de género, la supresión de la violencia como uno de los mecanismos de interacción, el contexto relacional familiar, y el mejoramiento de las condiciones de vulnerabilidad-pobreza. Esto se logrará con una mejor construcción de redes de apoyo social y políticas públicas que garanticen un acceso a la educación de calidad.

\section{Referencias}

Amone-P'Olak, K., Garnefski, N., \& Kraaij, V. (2007). The impact of war experiences and physical abuse on formerly abducted boys in northern Uganda: original article. South African Psychiatry Review, 10, 76-82.

Beck, N. I., Arif, I., Paumier, M. F., \& Jacobsen, K. H. (2016). Adolescent injuries in Argentina, Bolivia, Chile, and Uruguay: Results from the 2012-2013 Global School-based Student Health Survey (GSHS). Injury, 47, 2642-2649. doi:10.1016/j.injury.2016.10.002

Bonanno, R. A., y Hymel, S. (2010). Beyond hurt feelings: Investigating why some victims of bullying are at greater risk for suicidal ideation. Merrill-Palmer Quarterly, 56, 420-440.

Borowsky, I. W., Taliaferro, L. A., \& McMorris, B. J. (2013). Suicidal thinking and behavior among youth involved in verbal and social bullying: Risk and protective factors. Journal of adolescent health, 53, 4-12. doi: 10.1016/j.jadohealth.2012.10.280

Brook, J. S., Brook, D. W., \& Whiteman, M. (2007). Growing up in a violent society: longitudinal predictors of violence in Colombian adolescents. American journal of community psychology, 40, 82-95. doi: 10.1007/s10464-007-9126-Z

Cha, C. B., \& Nock, M. K. (2009). Emotional intelligence is a protective factor for suicidal behavior. Journal of the American Academy of Child and Adolescent Psychiatry, 48, 422-430. doi: 10.1097/ CHI.0b013e3181984f44.

Campo-Arias, A., \& Herazo, E. (2015). Asociación entre desigualdad y tasa de suicidio en Colombia (1994-2013). Revista Colombiana de Psiquiatría, 44, 28-32.

Castellví, P., Miranda-Mendizábal, A., Parés-Badell, O., Almenara, J., Alonso, I., Blasco, M. J. \& Piqueras, J. A. (2017). Exposure to vio- lence, a risk for suicide in youths and young adults. A meta analysis of longitudinal studies. Acta psychiatrica Scandinavica, 135, 195-211. doi: 10.1111/acps.12679

Chen, W. Y. (2010). Exposure to community violence and adolescents' internalizing behaviors among African American and Asian American adolescents. Journal of youth and adolescence, 39, 403-413. doi: 10.1007/s10964-009-9427-7

Cole, P. M. (2014). Moving ahead in the study of the development of emotion regulation. International Journal of Behavioral Development, 38, 203-207.

Czyz, E. K., Horwitz, A. G., Eisenberg, D., Kramer, A., \& King, C. A. (2013). Self-reported barriers to professional help seeking among college students at elevated risk for suicide. Journal of American College Health, 61, 398-406. doi: 10.1080/07448481.2013.820731

Daine, K., Hawton, K., Singaravelu, V., Stewart, A., Simkin, S., \& Montgomery, P. (2013). The power of the web: a systematic review of studies of the influence of the internet on self-harm and suicide in young people. PloS one, 8(10), e77555. doi:10.1371/journal.pone.0077555

Dapieve, N., \& Dalvosco, D. (2017). Prevalência de Exposição à Violência Direta e Indireta: um Estudo com Adolescentes de Colégios Públicos. Acta Colombiana de Psicología, 20, 101-111.

Dasgupta, S., \& Hazra, S. (2011). Does student's emotional intelligence play role in their suicidal ideation. Indian Journal of Community Psychology, 7, 190-197.

Debarbieux, E. (2003). School violence and globalisation. Journal of educational administration, 41, 582-602. doi:10.1108/09578230310504607

Devries, K. M., Mak, J. Y., Bacchus, L. J., Child, J. C., Falder, G., Petzold, M., ... \& Watts, C. H. (2013). Intimate partner violence and incident depressive symptoms and suicide attempts: a systematic review of longitudinal studies. PLoS Med, 10, e1001439. doi:10.1371/journal. pmed.1001439

Duncan, D. T., \& Hatzenbuehler, M. L. (2014). Lesbian, gay, bisexual, and transgender hate crimes and suicidality among a population-based sample of sexual-minority adolescents in Boston. American journal of public health, 104, 272-278. doi: 10.2105/AJPH.2013.301424.

Dunlop, S. M., More, E., \& Romer, D. (2011). Where do youth learn about suicides on the Internet, and what influence does this have on suicidal ideation? Journal of child psychology and psychiatry, 52, 1073-1080. doi: 10.1111/j.1469-7610.2011.02416.x

Espinoza-Gómez, F., Zepeda-Pamplona, V., Bautista-Hernández, V., Hernández-Suárez, C., Newton-Sánchez, O., \& Placensia-García, G. (2010). Violencia doméstica y riesgo de conducta suicida en universitarios adolescentes. Salud Pública México, 52, 213-219.

Ford, J. D., Elhai, J. D., Connor, D. F., \& Frueh, B. C. (2010). Poly-victimization and risk of posttraumatic, depressive, and substance use disorders and involvement in delinquency in a national sample of adolescents. Journal of Adolescent Health, 46, 545-552. Doi: 10.1016/j. jadohealth.2009.11.212

Ford, J. D., Hartman, J. K., Hawke, J., \& Chapman, J. F. (2008). Traumatic victimization, posttraumatic stress disorder, suicidal ideation, and substance abuse risk among juvenile justice-involved youth. Journal of Childy Adolescent Trauma, 1, 75-92. Doi: 10.1080/19361520801934456 
Gámez-Guadix, M., \& Calvete, E. (2012). Violencia filioparental y su asociación con la exposición a la violencia marital y la agresión de padres a hijos. Psicothema, 24, 277-283.

Guedes, A., García-Moreno, C., \& Bott, S. (2014). Violencia contra las mujeres en Latinoamérica y el Caribe. Foreign Affairs Latinoamérica, 14, 41-8.

Hagan, C. R., \& Joiner, T. E. (2016). The indirect effect of perceived criticism on suicide ideation and attempts. Archives of Suicide Research, 1-17. doi:10.1080/13811118.2016.1218398

Haw, C., Hawton, K., Niedzwiedz, C., \& Platt, S. (2013). Suicide clusters: a review of risk factors and mechanisms. Suicide and life-threatening behavior, 43, 97-108. doi: 10.1111/j.1943-278x.2012.00130.x

Hawton, K., Saunders, K. E., \& O'Connor, R. C. (2012). Self-harm and suicide in adolescents. The Lancet, 379, 2373-2382. doi: 10.1016/ S0140-6736(12)60322-5

Hewitt, N., Gantiva, C. A., Vera, A., Cuervo, M. P., Hernández, N. L., Juárez, F., \& Parada, A. J. (2014). Afectaciones psicológicas de niños y adolescentes expuestos al conflicto armado en una zona rural de Colombia. Acta Colombiana de Psicología, 17, 79-89. doi: 10.14718/ ACP.2014.17.1.9

Hinduja, S., \& Patchin, J. W. (2010). Bullying, cyberbullying, and suicide. Archives of suicide research, 14, 206-221. doi:10.1080/1381111 8.2010 .494133

Hooven, C., Nurius, P. S., Logan-Greene, P., \& Thompson, E. A. (2012). Childhood violence exposure: Cumulative and specific effects on adult mental health. Journal of family violence, 27, 511-522. doi: 10.1007/ s10896-012-9438-0

Hoyos de los Ríos, O. L., Llanos Martínez, M., \& Valega Mackenzie, S. J. (2012). El maltrato entre iguales por abuso de poder en el contexto universitario: incidencia, manifestaciones y estrategias de solución. Universitas Psychologica, 11, 793-802.

Hoyos, O., Aparicio, J., \& Córdoba, P. (2005). Caracterización del maltrato entre iguales en una muestra de colegios de Barranquilla (Colombia). Psicología desde el Caribe, 1-28.

Instituto de Medicina Legal y Ciencias Forenses. (2013). Comportamiento del homicidio. Colombia, 2013. Recuperado de http://www.medicinalegal.gov.co/documents/10180/188820/FORENSIS+2013+2-+homicidio. pdf/2af79b03-2a12-4341-a9a7-c3d9a251c38f

Instituto de Medicina Legal y Ciencias Forenses. (2015a). Comportamiento del homicidio. Colombia, 2015. Recuperado desde http://www. medicinalegal.gov.co/documents/88730/3418907/2.+HOMICIDIOS. pdf/70a4c34b-920c-465b-9902-936ffeab4afd

Instituto de Medicina Legal y Ciencias Forenses. (2015b). Comportamiento de la Violencia Intrafamiliar. Colombia, 2015. Recuperado desde http://www.medicinalegal.gov.co/documents/88730/3418907/4.+VIOLENCIA+INTRAFAMILIAR-+1+parte.pdf/57a54b37-954e-4c848 fec-ab2566a592b6

Jacobson, C. M., Marrocco, F., Kleinman, M., y Gould, M. S. (2011). Restrictive emotionality, depressive symptoms, and suicidal thoughts and behaviors among high school students. Journal of Youth and Adolescence, 40, 656-665. doi: 10.1002/jts.20116

Joiner Jr, T. E., Brown, J. S., \& Wingate, L. R. (2005). The psychology and neurobiology of suicidal behavior. Annu. Rev. Psychol, 56, 287-314.

Kim, J., \& Cicchetti, D. (2010). Longitudinal pathways linking child maltreatment, emotion regulation, peer relations, and psychopathology. Journal of Child Psychology and Psychiatry, 51, 706-716. doi: 10.1111/j.1469-7610.2009.02202.x

Klomek, A. B., Sourander, A., \& Gould, M. (2010). The association of suicide and bullying in childhood to young adulthood: a review of cross-sectional and longitudinal research findings. The Canadian Journal of Psychiatry, 55, 282-288.

Lambert, S. F., Boyd, R. C., Cammack, N. L., \& Ialongo, N. S. (2012). Relationship proximity to victims of witnessed community violence: associations with adolescent internalizing and externalizing behaviors. American journal of orthopsychiatry, 82, 1.doi: 10.1111/j.19390025.2011.01135.x

Lipschitz, J. M., Yen, S., Weinstock, L. M., \& Spirito, A. (2012). Adolescent and caregiver perception of family functioning: Relation to suicide ideation and attempts. Psychiatry research, 200, 400-403. doi: 10.1016/j.psychres.2012.07.051.

Lowry, R., Crosby, A. E., Brener, N. D., \& Kann, L. (2014). Suicidal thoughts and attempts among US high school students: trends and associated health-risk behaviors, 1991-2011. Journal of Adolescent Health, 54(1), 100-108. doi: 10.1016/j.jadohealth.2013.07.024.

Massey, S. H., Compton, M. T., \& Kaslow, N. J. (2014). Attachment security and problematic substance use in low-income, suicidal, African American women. The American Journal on Addictions, 23, 294-299.

Mandal, E., \& Zalewska, K. (2010). Psychiczna kobiecość i męskość, poczucie własnej atrakcyjności, style przywiązania, style radzenia sobie i strategie autoprezentacji u kobiet podejmujących próby samobójcze. Psychiatria polska, 44(3), 329-339.

Mandal, E., \& Zalewska, K. (2012). Style przywiązania, traumatyczne doświadczenia z okresu dzieciństwa i dorosłości, stany psychiczne oraz metody podejmowania prób samobójczych przez kobiety leczone psychiatrycznie. Psychiatr. Pol, 46, 75-84.

Margolin, G., \& Vickerman, K. A. (2007). Posttraumatic stress in children and adolescents exposed to family violence: I. Overview and issues. Professional Psychology: Research and Practice, 38, 613. doi: 10.1037/0735-7028.38.6.613

Mejia, R., Kliewer, W., \& Williams, L. (2006). Domestic violence exposure in Colombian adolescents: Pathways to violent and prosocial behavior. Journal of traumatic stress, 19, 257-267. Doi: 10.1002/jts.20116

Mels, C., \& Fernández, L. (2015). Violencia comunitaria en adolescentes desfavorecidos: exposición, impacto percibido y consecuencias psicológicas. Revista de Psicología, 24. doi: 10.5354/0719-0581.2015.36863

Messias, E., Castro, J., Saini, A., Usman, M., \& Peeples, D. (2011). Sadness, suicide, and their association with video game and internet overuse among teens: results from the youth risk behavior survey 2007 and 2009. Suicide and Life-Threatening Behavior, 41, 307-315. doi: 10.1111/j.1943-278X.2011.00030.x

Mrug, S., \& Windle, M. (2010). Prospective effects of violence exposure across multiple contexts on early adolescents' internalizing and externalizing problems. Journal of Child Psychology and Psychiatry, 51, 953-961. doi: 10.1111/j.1469-7610.2010.02222.x 
Navarro-Gómez, N. (2017). El suicidio en jóvenes en España: cifras y posibles causas. Análisis de los últimos datos disponibles. Clínica y Salud, 28, 25-31. doi: 10.1016/j.clysa.2016.11.002

Nikolaev, E., Baranojva, E., \& Petunova, S. (2016). Problemas de salud mental en niños pequeños: las estrategias de afrontamiento y estilos de crianza y características de funcionamiento de la familia Madres. Procedia-Ciencias Sociales y Del Comportamiento, 17, 94-99. doi:10.1016/j.sbspro.2016.10.152

Orue, I. (2010). Elaboración y validación de un cuestionario para medir la exposición a la violencia en infancia y adolescencia. Revista Internacional de Psicología y Terapia Psicológica, 10, 279-292.

Ozer, E. J. (2005). The impact of violence on urban adolescents: Longitudinal effects of perceived school connection and family support. Journal of Adolescent Research, 20, 167-192.

Pardo, M. E., Pineda, S., Carrillo, S., \& Castro, J. (2006). Análisis psicométrico del inventario de apego con padres y pares en una muestra de adolescentes colombianos. Interamerican Journal of Psychology, 40, 289-302.

Piedrahita, S., Elvira, L., Martinez, D. A., \& Vinazco, E. L. (2007). Significado de la violencia en niños de 6 a 12 años de una institución educativa perteneciente al sector oficial. Universitas psychologica, 6, 581-588.

Pirkis, J., y Blood, W. (2010). Suicide and the news and information media: A critical review. Retrieved desde http:// www.mindframe-media.info/ site $/$ index.cfm?display $=84378$.

Pisani, A. R., Wyman, P. A., Petrova, M., Schmeelk-Cone, K., Goldston, D. B., Xia, Y., \& Gould, M. S. (2013). Emotion regulation difficulties, youth-adult relationships, and suicide attempts among high school students in underserved communities. Journal of youth and adolescence, 42, 807-820. doi: 10.1007/s10964-012-9884-2

Plutchick, R., Van Praga, HM., Conte, HR. \& Picard, S. (1989). Correlates of Suicide and Violence Risk1: The Suicide Risk Measure. Comprehensive Psychiatry, 30, 296-302.

Rajalin, M., Hirvikoski, T., \& Jokinen, J. (2013). Family history of suicide and exposure to interpersonal violence in childhood predict suicide in male suicide attempters. Journal of affective disorders, 148, 92-97. doi:10.1016/j.jad.2012.11.055

Rajalin, M., Hirvikoski, T., \& Jokinen, J. (2013). Family history of suicide and exposure to interpersonal violence in childhood predict suicide in male suicide attempters. Journal of affective disorders, 148, 92-97. doi:10.1016/j.jad.2012.11.055

Ramírez, C. (2006). El impacto del maltrato en los niños y las niñas en Colombia. Revista infancia, adolescencia y familia, 1, 287-301.

Raver, C. C. (2004). Placing emotional self regulation in sociocultural and socioeconomic contexts. Child development, 75, 346-353. doi: 10.1111/j.1467-8624.2004.00676.x

Ray, M., \& Jat, K. R. (2010). Effect of electronic media on children. Indian pediatrics, 47, 561-568. doi: 10.1007/s13312-010-0128-9

Rigby, K., \& Slee, P. (1999). Suicidal ideation among adolescent school children, involvement in bully-victim problems, and perceived social support. Suicide and life-threatening behavior, 29, 119-130. doi: 10.1111/j.1943-278X.1999.tb01050.x
Rojas, V. (2008). Influencia de la televisión y videojuegos en el aprendizaje y conducta infanto-juvenil. Revista chilena de pediatría, 79, 81-85. doi: 10.4067/S0370-41062008000700012

Rubio, G, Montero, I., Jáuregui, J., Villanueva, R., Casado, M. A., Marín, J. J., \& Santo Domingo J. (1998). Validación de la escala de riesgo suicida de Plutchik en población española. Arch Neurobiol, 61, 143-152.

Sandoval Escobar, M. (2006). Los efectos de la televisión sobre el comportamiento de las audiencias jóvenes desde la perspectiva de la convergencia y de las prácticas culturales. Universitas Psychologica, 5, 205-222.

Savin-Williams, R. C. (1994). Verbal and physical abuse as stressors in the lives of lesbian, gay male, and bisexual youths: associations with school problems, running away, substance abuse, prostitution, and suicide. Journal of consulting and clinical psychology, 62, 261. Doi:10.1037/0022-006X.62.2.261

Strenziok, M., Krueger, F., Pulaski, S. J., Openshaw, A. E., Zamboni, G., Van der Meer, E., \& Grafman, J. (2010). Lower lateral orbitofrontal cortex density associated with more frequent exposure to television and movie violence in male adolescents. Journal of Adolescent Health, 46, 607-609. Doi: 10.1016/j.jadohealth.2009.11.196

Suárez, Y., Restrepo, D., \& Caballero, C. (2016). Ideación suicida y su relación con la inteligencia emocional en universitarios colombianos. Revista Salud Uis, 48, 470-478. doi: 10.18273/revsalv4n4-2016005

Suárez, Y., \& Wilches, C. (2015). Habilidades emocionales en una muestra de estudiantes universitarios: las diferencias de género. Educación y Humanismo, 17, 119-132. doi:10.17081/eduhum.17.28.1170

Swahn, M. H., Gaylor, E., Bossarte, R. M., \& Dulmen, M. V. (2010). Co-occurring suicide attempts and physical fighting: a comparison between urban, suburban, and rural high school students. Vulnerable children and youth studies, 5, 353-362. doi:1080/17450128.2010.516373

Takizawa, R., Maughan, B., \& Arseneault, L. (2014). Adult health outcomes of childhood bullying victimization: evidence from a five-decade longitudinal British birth cohort. American journal of psychiatry, 171, 777-784. doi: 10.1176/appi.ajp.2014.13101401

Vermeiren, R., Ruchkin, V., Leckman, P. E., Deboutte, D., \& Schwab-Stone, M. (2002). Exposure to violence and suicide risk in adolescents: A community study. Journal of abnormal child psychology, 30, 529-537. doi:10.1023/A:1019825132432

Wadsworth, M. E., \& Berger, L. E. (2006). Adolescents coping with poverty-related family stress: Prospective predictors of coping and psychological symptoms. Journal of Youth and Adolescence, 35, 5467. doi: 10.1007/s10964-005-9022-5

Wadsworth, M. E., \& Compas, B. E. (2002). Coping with family conflict and economic strain: The adolescent perspective. Journal of Research on Adolescence, 12, 243-274. doi: 10.1111/1532-7795.00033

Waldrop, A. E., Hanson, R. F., Resnick, H. S., Kilpatrick, D. G., Naugle, A. E., \& Saunders, B. E. (2007). Risk factors for suicidal behavior among a national sample of adolescents: Implications for prevention. Journal of traumatic Stress, 20(5), 869-879. doi:10.1002/jts.20291

Wang, Y., Sareen, J., Afifi, T. O., Bolton, S. L., Johnson, E. A., \& Bolton, J. M. (2015). A population-based longitudinal study of recent stressful life events as risk factors for suicidal behavior in major 
depressive disorder. Archives of suicide research, 19, 202-217. Doi: 10.1080/13811118.2014.957448

Waseem, M., Ryan, M., Foster, C. B., \& Peterson, J. (2013). Assessment and management of bullied children in the emergency department. Pediatric emergency care, 29, 38. doi: 10.1097/PEC.0b013e31828575d7

Waseem, M., Ryan, M., Foster, C. B., \& Peterson, J. (2013). Assessment and management of bullied children in the emergency department. Pediatric emergency care, 29, 389. doi: 10.1097/PEC.0b013e31828575d7

Yip, P. S., Fu, K. W., Yang, K. C., Ip, B. Y., Chan, C. L., Chen, E. Y., Et Al. (2006). The effects of a celebrity suicide on suicide rates in Hong Kong. Journal of Affective Disorders, 93, 245-252. doi: 10.1016/j. jad.2006.03.015g 
